\title{
Multiple Physical Symptoms Are Useful to Identify High Risk Individuals for Burnout: A Study on Faculties and Hospital Workers in Japan
}

\author{
Yuki Chatani ${ }^{1}$, Kyoko Nomura ${ }^{2, *}$, Haruko Hiraike ${ }^{3} \mathbb{D}$, Akiko Tsuchiya ${ }^{4}$ and Hiroko Okinaga ${ }^{5}$ \\ 1 Department of Anesthesiology, National Hospital Organization Saitama Hospital, Saitama 351-0102, Japan; \\ iprofm_29682@yahoo.co.jp \\ 2 Department of Public Health, Akita University Graduate School of Medicine, Akita 010-8543, Japan \\ 3 Department of Obstetric and Gynecology, Teikyo University Itabashi Hospital, Tokyo 173-0003, Japan; \\ haruko.hiraike@gmail.com \\ 4 Division of Nursing, Teikyo University Itabashi Hospital, Tokyo 173-0003, Japan; a-tutiya@med.teikyo-u.ac.jp \\ 5 Support Center for Women Physicians and Researchers, Teikyo University, Tokyo 173-0003, Japan; \\ hiokinaga@gmail.com \\ * Correspondence: nomurakyoko@gmail.com; Tel.: +81-18-884-6087 or +81-18-884-6086
}

\section{check for}

updates

Citation: Chatani, Y.; Nomura, K.; Hiraike, H.; Tsuchiya, A.; Okinaga, $\mathrm{H}$. Multiple Physical Symptoms Are Useful to Identify High Risk Individuals for Burnout: A Study on Faculties and Hospital Workers in Japan. Int. J. Environ. Res. Public Health 2021, 18, 3246. https:// doi.org/10.3390/ijerph18063246

Academic Editors: Juan A. Moriano and Ana Laguía

Received: 21 February 2021

Accepted: 17 March 2021

Published: 21 March 2021

Publisher's Note: MDPI stays neutral with regard to jurisdictional claims in published maps and institutional affiliations.

Copyright: (c) 2021 by the authors. Licensee MDPI, Basel, Switzerland. This article is an open access article distributed under the terms and conditions of the Creative Commons Attribution (CC BY) license (https:// creativecommons.org/licenses/by/ $4.0 /)$.

\begin{abstract}
Healthcare workers have a high risk of burnout. This study aimed to investigate if the numbers of physical symptoms are associated with burnout among healthcare workers. We conducted a cross-sectional survey at a large university in Tokyo, Japan, in 2016. Participants were 1080: 525 faculties and 555 hospital workers. We investigated 16 physical symptoms perceived more than once per week and examined the association between the number of physical symptoms and Copenhagen Burnout Inventory (CBI); work-related (WBO), personal (PBO), and client-related (CBO) burnout. All CBI scores were higher among hospital workers than among faculties: WBO (43 vs. 29), PBO (50 vs. 33), CBO (33 vs. 29). Moreover, the higher the number of physical symptoms perceived, the higher the degree of burnout scores became (trend $p$-values $<0.001$ ), except for CBO among faculties. Job strain (all except for CBO among hospital workers) and work-family conflict were associated with an increased risk of burnout. Being married (WBO and CBO among faculties), having a child (except for PBO and CBO among faculties), and job support (faculty and hospital workers with WBO and faculties with PBO) were associated with a decreased risk of burnout. Multiple physical symptoms might be useful for identifying high risk individuals for burnout.
\end{abstract}

Keywords: physical symptoms; burnout; Copenhagen Burnout Inventory; hospital workers; faculties; work-family conflict

\section{Introduction}

During their daily clinical work, healthcare professionals sometimes encounter patients who report physical complaints—such as fatigue, backache, headache, and stomachachewith no apparent cause. Usually, they claim various kinds of physical symptoms but healthcare professionals often cannot find any underlying diseases. In such cases, we had the hypothesis that it can be due to mental health malfunction. There are a few studies to support our theory and one previous study suggested that patients with multiple unspecified physical complaints often have mental health problems [1,2]. Among previous studies that investigated the association between physical symptoms and mental health, one study on Latino and Asian Americans found that people with more physical symptoms with no apparent cause often seek mental health service [3].

Another study concurred with this theory and demonstrated that unspecified eye symptoms - such as blurred vision and trouble seeing without a specific diagnosis-are associated with worse self-perceived mental health [4]. Burnout is a mental health issue characterized by a state of emotional, physical, and mental exhaustion [5]. In this study, we 
focused on burnout among faculties and hospital workers; these individuals are particularly vulnerable to burnout, due to the daily stress of managing patients and coping with their lives and deaths, dealing with patients' families, and often working in conditions, including frequent night shifts and overtime. More recently, the situation may be even worse because of the COVID-19 pandemic, which causes faculties and hospital workers-including doctors and nurses - to experience intense psychological pressure [6]. Ignoring faculty and hospital workers' possible burnout may lead to them developing adverse health outcomes such as depression, which could, in turn, influence the healthcare system. In this regard, early intervention to address burnout benefits both workers and organizations, as high turnover rates-due to burnout - engender an additional financial burden to train new employees [7] as well as more extra personnel and overworked employees who remain in the workplace [8]. Previous studies emphasized burnout as highly prevalent among doctors $[9,10]$, nurses [11,12], and medical faculties [13]; however, there are very few informative and practical studies that address the early indicators of burnout among healthcare professionals. We previously demonstrated that, among healthy white-collar workers, the number of self-reported physical symptoms is significantly associated with job stress [14], as measured by the Job Content Questionnaire (JCQ) [15]. In this study, we applied this concept to medical professionals to examine whether the number of physical symptoms could be used as an early indicator of burnout. It is known from previous studies that medical professionals usually hesitate to seek help at the workplace because they believe that disclosure of mental illness will harm their professional career [16]. If multiple physical symptoms are useful indicators of burnout, the self-reporting of multiple physical symptoms may be a good alternative to direct intervention for burnout in this population. This study therefore aimed to investigate whether multiple physical symptoms are associated with burnout among faculties at a medical university and hospital workers.

\section{Materials and Methods}

\subsection{Participants}

This cross-sectional study was a part of a university survey entitled "A Survey about Raising Children, Caregiving, Work Environment and Satisfaction of Work" which investigated work-life balance of faculties and hospital workers at a large private university in Japan. This university has five campuses that include schools of medicine, pharmacology, medical technology, science and engineering, economics, law, liberal arts, language, and education. At the time of the survey, two-thirds of the faculties belonged to medical-affiliated campuses with three affiliated hospitals; there were 1137 faculties (women: 20\%; School of Medicine: 70\%) and 2629 hospital workers registered at the three affiliated hospitals (women, 48\%). All of these faculties and hospital workers were invited to participate in this study in February 2016. A self-administered questionnaire and informed consent format were sent by post via the administrative office at each campus. The faculties comprised academic personnel-full professors as well as associated and assistant professors-who were employed by the university. A total of 1186 respondents provided consent and completed the self-administered questionnaire (response rate: $31 \%$ ). We excluded questionnaires with missing values on the 16 physical symptoms $(n=42)$, three types of the Copenhagen Burnout Inventory (CBI) scores $(n=20)$, whether they are faculties or hospital workers $(n=14)$, and gender $(n=30)$. The final sample comprised 1080 participants, including 525 faculties (women: 42\%) and 555 hospital workers (women: 88\%) who were included in the analyses.

We have followed the ethical standards of the institution and the 1964 Helsinki declaration and its later amendments or ethical standards in conducting this research. This study was approved by the ethics committee in Teikyo University (\#TEIRIN 15-141 in 2015). 


\subsection{Measures}

\subsubsection{Burnout}

The primary outcome of this study was the CBI $[17,18]$. The CBI measures workrelated burnout (WBO, 7 items), personal burnout (PBO, 6 items), and client-related burnout (CBO, 6 items). For this study, "clients" refer to students for faculties and to patients for hospital workers. A total of 19 questions were asked. Answers were presented on a 5-point Likert scale ranging from 100 points for "always/to a very high degree" to 0 points for "never/to a very low degree." If fewer than three questions were answered in the personal and patient-related burnout subscales or fewer than four questions in the work-related burnout subscale, the respondent was classified as a non-responder. The three burnout subscale scores were calculated with one item in reversed order relative to the response; therefore, higher overall scores indicate a higher degree of burnout.

Previous research has demonstrated that all three domains have high internal reliability, particularly among human service workers such as employees at hospitals [19], facilities for people with severe disabilities, and home care services [20]. Existing research also shows that all three burnout domains are associated with increased work absenteeism and predicts intention to leave the workplace [20,21].

\subsubsection{Physical Symptoms Perceived}

The main exposure variable was the number of self-reported physical symptoms. The 16 physical symptoms included in the questionnaire were headache, eye symptoms, dizziness, nausea, vomiting, diarrhea, constipation, abdominal pain, musculoskeletal pain, articular pain, backache, chest pain, palpitations, dyspnea, insomnia, and fatigue [22,23]. The frequency of these symptoms had 9 possible answers: "almost none", "less than once a month", "more than once a month", "once a week", "a few times a week", "4-6 times a week", "every day", "more than once a day", and "always". We defined physical symptoms as those experienced "once a week or more" (i.e., 1 vs. 0 ) and summed up the number of symptoms experienced. Based on the median number of $2(25-75 \%, 0-4)$, the total number was grouped into quartiles (i.e., $0 / 1-2 / 3-4 / 5-$ ).

\subsubsection{Work-Family Conflict (WFC)}

The Work-Family Conflict Scale used in this study was developed by Greenhaus [24] and Carlson [25] and then translated into Japanese [26]. This study uses one question from each of the 6 components of the WFC scale (time-based work interference with family, time-based family interference with work, strain-based work interference with family, strain-based family interference with work, behavior-based work interference with family, behavior-based family interference with work). These questions are as follows: (1) The time I must devote to my job keeps me from participating equally in household responsibilities and activities; (2) The time I spend on family responsibilities often interferes with my work activities; (3) When I get home from work, I am often too frazzled to participate in family activities/responsibilities; (4) Because I am often stressed due to family responsibilities, I have a hard time concentrating on my work; (5) The effective behaviors I perform do not help me to be a better parent and spouse; (6) The behaviors that work for me at home do not seem to be effective at work.

Responses were measured using a 5-point Likert scale from "most agree" (5) to "least agree" (1). The sum of these six items became a continuous variable expressing the degree of WFC.

\subsubsection{Work Stress and Social Support}

The Job Content Questionnaire (JCQ) [14] is a valid and reliable questionnaire to measure psychological stress and social support in the workplace [27]. Job strain was defined based on the Karasek model, which divides job demand by job control. The Karasek demand-control model is a well-known and established scientific measure for quantifying psychological stress at work. The conceptual idea of job strain is that workplace 
stress is a function of how demanding a person's job is and how much control the person has over their responsibilities in terms of discretion, authority, or decision latitude. High strain was defined as $>75$ percentile and low strain as the $\leq 75$ percentile. Social support was defined as high support ( $>50$ percentile) and low support ( $\leq 50$ percentile).

\subsubsection{Covariates}

Other items investigated in this study included age group (i.e., 20s, 30s, 40s, or older), marital status (i.e., married, single, divorced, or widowed), presence of a child or not, housekeeping hours on weekdays (i.e., $1.5 \mathrm{~h}$ or more and $1.5 \mathrm{~h}$ or less) and weekends (i.e., $\geq 3 \mathrm{~h}$ or $<3 \mathrm{~h}$ ), faculty worker at university or university-affiliated hospital worker, years of work experience, work hours, working nights per month (i.e., $\geq 3.5$ nights or $<3.5$ nights), and job categories (i.e., medical doctor, pharmacist, nurse, medical engineer, or other).

\subsection{Statistical Analyses}

Variables were statistically investigated to determine if they differed according to the profession group (faculty or hospital worker). Statistical significance was determined using a chi-squared test for categorical variables and a $t$-test or Wilcoxon test for continuous variables. General linear models with the outcome of three subtypes of the CBI subscale scores were applied to investigate whether multiple physical symptoms were associated with the CBI score. Simultaneous regression model was used to determine which variables were significant. Trend $P$ for the linearity of the quartile of the total numbers was estimated in the final multivariable models. Sensitivity analyses were performed to determine how much the following particular symptoms are associated with burnout degree: fatigue, backache, and insomnia. These specific symptoms were chosen as the former two are the most frequent symptoms and insomnia is a risk factor for depression [28]. The statistical interactions between any significant variables were investigated in the final regression models.

The significance level was set at $p<0.050$. All analyses were performed using SAS software (version 9.4; SAS Institute, Cary, NC, USA).

\section{Results}

\subsection{Baseline Characterstics and Working Conditions}

Table 1 shows the baseline characteristics and working conditions according to profession group. The median number (25-75\%) of physical symptoms was $1.0(0-3)$ among faculties and $2.0(1-5)$ among hospital workers $(p<0.001)$. Of the women, the occupation with the highest percentage was nurses (79\%), while among men, most $(59 \%)$ were doctors. Hospital workers were more likely to be women, single, in their 20s, work longer hours at home on weekdays, and have WFC. The majority of faculties were men, aged $\geq 40$ years, married, and more likely to have a child. Hospital workers perceived higher job strain than faculties did $(p<0.001)$ and had higher burnout scores in all three domains (WBO: 43 vs. 29; PBO: 50 vs. 33 ; CBO: 33 vs. 29 , all $p$-values $<0.001$ ). 
Table 1. Baseline characteristics and working conditions according to profession.

\begin{tabular}{|c|c|c|c|c|}
\hline & $\begin{array}{l}\text { Total } n=1080 \\
\text { Percent }(\%)\end{array}$ & $\begin{array}{l}\text { Faculty } n=555 \\
\text { Percent }(\%)\end{array}$ & $\begin{array}{c}\text { Hospital Worker } \\
n=525 \text { Percent }(\%)\end{array}$ & $p$-Value \\
\hline Gender & & & & $<0.001$ \\
\hline Women & 65 & 42 & 88 & \\
\hline Men & 35 & 58 & 12 & \\
\hline Age group & & & & $<0.001$ \\
\hline $20 \mathrm{~s}$ & 35 & 17 & 52 & \\
\hline $30 \mathrm{~s}$ & 22 & 21 & 23 & \\
\hline 40 s or older & 43 & 62 & 25 & \\
\hline Marital status & & & & $<0.001$ \\
\hline Married & 50 & 66 & 35 & \\
\hline Single & 50 & 34 & 65 & \\
\hline Presence of a child & & & & $<0.001$ \\
\hline Yes & 43 & 55 & 33 & \\
\hline No & 57 & 45 & 67 & \\
\hline $\begin{array}{l}\text { Housekeeping hours in weekdays, } \\
\text { median }(25 \%, 75 \%)\end{array}$ & $1.5(1.0,3.0)$ & $1.0(0.5,2.0)$ & $2.0(1.0,3.0)$ & $<0.001$ \\
\hline $\begin{array}{l}\text { Housekeeping hours in weekend, } \\
\text { median }(25 \%, 75 \%)\end{array}$ & $3.0(2.0,5.0)$ & $3.0(1.5,4.0)$ & $3.0(2.0,5.0)$ & 0.330 \\
\hline $\begin{array}{l}\text { Work Family Conflict, median } \\
\qquad(25 \%, 75 \%)\end{array}$ & $17(14,19)$ & $16(13,18)$ & $18(15,20)$ & $<0.001$ \\
\hline $\begin{array}{l}\text { Working Experience years, median } \\
(25 \%, 75 \%)\end{array}$ & $9.0(3.0,19)$ & $11(5.0,22)$ & $6.0(3.0,15)$ & $<0.001$ \\
\hline Work hours, median $(25 \%, 75 \%)$ & $9.0(8.0,10)$ & $9.0(8.0,11)$ & $9.0(8.0,10)$ & $<0.001$ \\
\hline $\begin{array}{l}\text { Working nights per month, median } \\
\qquad(25 \%, 75 \%)\end{array}$ & $3.0(0,5.0)$ & $2.0(0,4.0)$ & $3.0(0,5.0)$ & 0.002 \\
\hline Job strain & & & & $<0.001$ \\
\hline High strain & 25 & 15 & 33 & \\
\hline Low strain & 75 & 85 & 67 & \\
\hline Support & & & & 0.510 \\
\hline High support & 53 & 54 & 52 & \\
\hline Low support & 47 & 46 & 48 & \\
\hline $\begin{array}{l}\text { Physical symptom median, } \\
\qquad(25 \%, 75 \%)\end{array}$ & $2.0(0,4.0)$ & $1.0(0,3.0)$ & $2.0(1.0,5.0)$ & $<0.001$ \\
\hline \multicolumn{5}{|l|}{ Burnout, median $(25 \%, 75 \%)$} \\
\hline Work-related & $36(21,50)$ & $29(18,43)$ & $43(24,54)$ & $<0.001$ \\
\hline Personal & $42(25,58)$ & $33(21,50)$ & $50(33,67)$ & $<0.001$ \\
\hline Client-related & $33(21,46)$ & $29(21,42)$ & $33(21,50)$ & $<0.001$ \\
\hline Job category & & & & $<0.001$ \\
\hline Doctor & 19 & 23 & 4.0 & \\
\hline Pharmacist & 3.1 & 1.0 & 3.6 & \\
\hline Nurse & 65 & 21 & 6 & \\
\hline Engineer & 13 & 3.2 & 17 & \\
\hline Other & 25 & 48 & 0 & \\
\hline
\end{tabular}

\subsection{Physical Symptoms}

Table 2 shows the physical symptoms perceived once per week or more by profession. Of the 16 symptoms, the most frequent complaint was fatigue ( $55 \%$ ), followed by backache $(37 \%)$. These two symptoms as well as insomnia were observed more frequently in hospital workers than in faculties $(p<0.001$ for fatigue, $p=0.023$ for backache, and $p<0.001$ for insomnia). 
Table 2. Physical symptoms perceived once in a week or more by profession.

\begin{tabular}{ccccc}
\hline & Total $(\boldsymbol{n}=\mathbf{1 0 8 0})$ & Faculty $(\boldsymbol{n}=\mathbf{5 5 5})$ & Hospital Worker $(\boldsymbol{n}=\mathbf{5 2 5})$ & $p$-Value \\
\hline Fatigue & $\mathbf{\%}$ & $\mathbf{\%}$ & $\mathbf{\%}$ & \\
Backache & 55 & 49 & 61 & $<0.001$ \\
Eye symptom & 37 & 33 & 40 & 0.023 \\
Headache & 24 & 24 & 25 & 0.690 \\
Constipation & 21 & 16 & 25 & $<0.001$ \\
Insomnia & 20 & 16 & 23 & $<0.001$ \\
Diarrhea & 17 & 14 & 21 & $<0.001$ \\
Abdominal pain & 12 & 11 & 13 & 0.300 \\
Tinnitus & 12 & 7.6 & 16 & $<0.001$ \\
Arthritis & 11 & 10 & 11 & 0.510 \\
Myalgia & 10 & 9.1 & 9.2 & 0.190 \\
Shortness of breath & 9.4 & 9.1 & 9.7 & 0.740 \\
Palpitation & 9.1 & 6.9 & 9.1 & 0.100 \\
Dizziness & 7.9 & 5.3 & 8.8 & $<0.001$ \\
Nausea & 7.1 & 4.6 & 8.8 & 0.030 \\
Chest pain & 6.9 & 3.6 & 9.2 & $<0.001$ \\
\hline
\end{tabular}

\subsection{The Results of the General Linear Models}

\subsubsection{Factors Associated with $\mathrm{WBO}, \mathrm{PBO}$ and $\mathrm{CBO}$ among Faculties}

Table 3 shows the results of the univariable general linear models for factors associated with $\mathrm{WBO}, \mathrm{PBO}$, and $\mathrm{CBO}$ among faculties. Almost all the covariates were significant except for gender (only $\mathrm{CBO}$ ), housekeeping hours in weekdays (only $\mathrm{CBO}$ ), housekeeping hours in weekends (only WBO and $\mathrm{CBO}$ ), working experiences and working nights per month.

Table 3. Univariable models of an effect of covariates according to burnout subscale among faculties.

\begin{tabular}{|c|c|c|c|c|c|c|c|c|c|}
\hline & \multicolumn{3}{|c|}{ WBO } & \multicolumn{3}{|c|}{ PBO } & \multicolumn{3}{|c|}{ CBO } \\
\hline & $\beta$ & SE & $p$-Value & $\beta$ & SE & $p$-Value & $\beta$ & SE & $p$-Value \\
\hline Women vs. men & 8.6 & 1.6 & $<0.001$ & 12 & 1.9 & $<0.001$ & 1.5 & 1.6 & 0.343 \\
\hline Age group vs. 40 s or older & & & $<0.001 \mathrm{~d}$ & & & $<0.001 \mathrm{~d}$ & & & $0.007 \mathrm{~d}$ \\
\hline $20 s$ & 13 & 2.1 & & 14 & 2.6 & & 4.8 & 2.1 & \\
\hline $30 s$ & 5.1 & 2.0 & & 5.5 & 2.4 & & 4.3 & 1.9 & \\
\hline Marital status (Married vs. Single) & -13 & 1.6 & $<0.001$ & -13 & 1.9 & $<0.001$ & -7.4 & 1.6 & $<0.001$ \\
\hline Presence of a child & -11 & 1.6 & $<0.001$ & -11 & 1.9 & $<0.001$ & -6.4 & 1.6 & $<0.001$ \\
\hline Housekeeping hours in weekdays & 4.0 & 1.6 & 0.014 & 7.7 & 1.9 & $<0.001$ & 0.41 & 1.5 & 0.790 \\
\hline Housekeeping hours in weekend & 2.0 & 1.6 & 0.219 & 5.0 & 1.9 & 0.009 & 0.60 & 1.5 & 0.693 \\
\hline WFC & 2.2 & 0.17 & $<0.001$ & 2.5 & 0.21 & $<0.001$ & 1.8 & 0.17 & $<0.001$ \\
\hline Working experience (years) & -3.2 & 1.7 & 0.053 & -1.6 & 2.0 & 0.415 & -2.1 & 1.6 & 0.191 \\
\hline Work hours & 1.3 & 0.39 & 0.001 & 1.4 & 0.46 & 0.002 & 1.3 & 0.37 & $<0.001$ \\
\hline Working nights per month & 4.1 & 2.2 & 0.061 & 4.1 & 2.7 & 0.134 & 2.9 & 2.2 & 0.183 \\
\hline High strain vs. Low strain & 23 & 2 & $<0.001$ & 26 & 2.4 & $<0.001$ & 15 & 2.0 & $<0.001$ \\
\hline High support vs. Low support & -8.0 & 1.6 & $<0.001$ & -11 & 1.8 & $<0.001$ & -5.1 & 1.5 & $<0.001$ \\
\hline Physical symptoms (Reference: none) & & & $<0.001 \mathrm{~d}$ & & & $<0.001 \mathrm{~d}$ & & & $<0.001 \mathrm{~d}$ \\
\hline $1-2$ & 8.8 & 1.8 & & 13 & 2.1 & & 6.7 & 1.9 & \\
\hline $3-4$ & 15 & 2.1 & & 22 & 2.3 & & 7.3 & 2.1 & \\
\hline $5-$ & 21 & 2.2 & & 31 & 2.4 & & 9.1 & 2.2 & \\
\hline \multicolumn{10}{|c|}{ Individual models for three particular symptoms } \\
\hline Fatigue & 16 & 1.4 & $<0.001$ & 23 & 1.6 & $<0.001$ & 8.2 & 1.5 & $<0.001$ \\
\hline Backache & 12 & 1.6 & $<0.001$ & 16 & 1.9 & $<0.001$ & 4.3 & 1.6 & 0.008 \\
\hline Insomnia & 15 & 2.2 & $<0.001$ & 20 & 2.6 & $<0.001$ & 7.2 & 2.2 & 0.001 \\
\hline
\end{tabular}

d trend $P$

WBO: work-related burnout PBO: personal burnout CBO: client-related burnout WFC: work-family conflict.

Table 4 shows the multivariable general linear model of WBO, $\mathrm{PBO}, \mathrm{CBO}$ among faculties. We excluded the working nights per month from covariates because more than half were missing values. 
Table 4. Multivariable general linear models of an effect of covariates according to burnout subscale among faculties.

\begin{tabular}{|c|c|c|c|c|c|c|c|c|c|c|}
\hline & & \multicolumn{3}{|c|}{ WBO } & \multicolumn{3}{|c|}{ PBO } & \multicolumn{3}{|c|}{ СВО } \\
\hline & & \multicolumn{3}{|c|}{$(n=462, \mathrm{R} 249 \%)$} & \multicolumn{3}{|c|}{$(n=462, \mathrm{R} 251 \%)$} & \multicolumn{3}{|c|}{$(n=462, \mathrm{R} 226 \%)$} \\
\hline & & $\beta$ & SE & $p$-Value & $\beta$ & SE & $p$-Value & $\beta$ & SE & $p$-Value \\
\hline Women vs. Men & & 0.180 & 1.6 & 0.855 & 2.2 & 1.9 & 0.258 & 3.3 & 1.9 & 0.072 \\
\hline Age group vs. 40 s or older & & & & 0.171 & & & 0.072 & & & 0.532 \\
\hline & $20 \mathrm{~s}$ & 4.0 & 2.6 & & 6.2 & 3.1 & & -2.6 & 2.9 & \\
\hline & $30 \mathrm{~s}$ & 0.28 & 1.8 & & 0.39 & 2.1 & & -0.04 & 2.0 & \\
\hline Marital status (Married vs. Single) & & -4.3 & 1.9 & 0.027 & -3.0 & 2.3 & 0.186 & -4.8 & 2.2 & 0.031 \\
\hline Presence of a child & & -4.4 & 1.8 & 0.019 & -3.5 & 2.1 & 0.106 & -3.2 & 2.1 & 0.132 \\
\hline Housekeeping hours in week & ays & 1.3 & 1.8 & 0.514 & 4.1 & 2.1 & 0.048 & -1.2 & 2.0 & 0.562 \\
\hline Housekeeping hours in week & & -2.4 & 1.7 & 0.170 & -2.6 & 2.0 & 0.190 & 0.48 & 2.0 & 0.809 \\
\hline WFC & & 1.5 & 0.17 & $<0.001$ & 1.5 & 0.19 & $<0.001$ & 1.3 & 0.19 & $<0.001$ \\
\hline Working experience & & -0.45 & 1.7 & 0.920 & 0.77 & 2 & 0.703 & -1.9 & 2.0 & 0.335 \\
\hline Work hours & & 0.33 & 0.33 & 0.271 & 0.41 & 0.39 & 0.287 & 0.48 & 0.38 & 0.205 \\
\hline High strain vs. Low strain & & 12 & 2.0 & $<0.001$ & 12 & 2.3 & $<0.001$ & 9.6 & 2.2 & $<0.001$ \\
\hline High support vs. Low support & & -3.3 & 1.3 & 0.016 & -4.5 & 1.6 & 0.004 & -2.2 & 1.5 & 0.145 \\
\hline \multirow[t]{4}{*}{ Physical symptoms (Reference: none) } & 0 & - & & $<0.001 \mathrm{~d}$ & - & & $<0.001 \mathrm{~d}$ & & & $0.176 \mathrm{~d}$ \\
\hline & $1-2$ & 5.5 & 1.6 & & 9.3 & 1.8 & & 3.8 & 1.8 & \\
\hline & $3-4$ & 8.5 & 1.9 & & 15 & 2.2 & & 2.7 & 2.1 & \\
\hline & $5-$ & 13 & 2 & & 23 & 2.4 & & 3.1 & 2.3 & \\
\hline \multicolumn{11}{|c|}{ Individual models for three particular symptoms } \\
\hline & Fatigue a & 8.8 & 1.4 & $<0.001$ & 15 & 1.6 & $<0.001$ & 3.2 & 1.6 & 0.043 \\
\hline & Backache b & 7.0 & 1.5 & $<0.001$ & 9.5 & 1.8 & $<0.001$ & 0.85 & 1.60 & 0.603 \\
\hline & Insomnia c & 7.5 & 2.0 & $<0.001$ & 12 & 2.4 & $<0.001$ & 1.8 & 2.2 & 0.411 \\
\hline \multicolumn{11}{|c|}{ WBO: work-related burnout PBO: personal burnout CBO: client-related burnout WFC: work-family conflict } \\
\hline $\begin{array}{c}\text { a } n=462 \mathrm{R} 248 \%, n=462 \mathrm{R} 250 \%, \\
n=462 \mathrm{R} 226 \% \\
\mathrm{~b} n=462 \mathrm{R} 246 \%, n=462 \mathrm{R} 243 \%, \\
n=462 \mathrm{R} 225 \% \\
\text { c } n=462 \mathrm{R} 245 \%, n=462 \mathrm{R} 243 \%, \\
n=462 \mathrm{R} 225 \% \\
\text { d trend P }\end{array}$ & & & & & & & & & & \\
\hline
\end{tabular}

The WBO score increased with the quartile of physical symptoms (+5.5 points for 1-2 symptoms; +8.5 points for $3-4$ symptoms; 13 points for 5 or more symptoms, $p<0.001$ ), WFC (+1.5 points, $p<0.001)$, and job strain (+12 points, $p<0.001)$. The WBO score (beta estimates) decreased when participants were married ( -4.3 score, $p=0.027)$, when they had a child ( -4.4 score, $p=0.019)$, and when they had job support ( -3.3 score, $p=0.016$ ). When either fatigue, backache, or insomnia was entered into the model instead of physical symptom quartile, each symptom was significantly associated with an increased risk of WBO (all $p$ values $<0.001$ ), among which fatigue was most strongly correlated with WBO (+8.8 points, $p<0.001)$. The PBO score increased with the quartile of physical symptoms (+9.3 points for $1-2$ symptoms; +15 points for $3-4$ symptoms; +23 points for 5 or more symptoms, $p<0.001)$, WFC $(+1.5$ points, $p<0.001)$, job strain $(+12$ points, $p<0.001)$, and housekeeping hours in weekdays ( +4.1 points, $p=0.048)$. The $\mathrm{PBO}$ score decreased when participants had workplace support $(-4.5$ points, $p=0.004)$. When either fatigue, backache, or insomnia was entered into the model instead of physical symptom quartile, each symptom was significantly associated with an increased risk of PBO (all $p$ values $<0.001)$, among which fatigue was most strongly correlated with $\mathrm{PBO}(+15$ points, $p<0.001)$.

The CBO score increased with WFC $(+1.3$ points, $p<0.001)$, and job strain $(+9.6$ points, $p<0.001$ ). The $\mathrm{CBO}$ score (beta estimates) decreased when participants were married $(-4.8$ score, $p=0.031)$. The quartile of physical symptoms, backache and insomnia was insignificant while fatigue became significant $(p=0.043)$. 


\subsubsection{Factors Associated with $\mathrm{WBO}, \mathrm{PBO}$ and $\mathrm{CBO}$ among Hospital Workers}

Table 5 shows the results of the univariable general linear models for factors associated with WBO, $\mathrm{PBO}$, and $\mathrm{CBO}$ among hospital workers. All the covariates were significant except for housekeeping hours in weekdays and weekends (only $\mathrm{PBO}$ ), gender and housekeeping hours in weekdays (only CBO).

Table 5. Univariable models of an effect of covariates according to burnout subscale among hospital workers.

\begin{tabular}{|c|c|c|c|c|c|c|c|c|c|}
\hline & \multicolumn{3}{|c|}{ WBO } & \multicolumn{3}{|c|}{ PBO } & \multicolumn{3}{|c|}{ СВО } \\
\hline & $\beta$ & SE & $p$-Value & $\beta$ & SE & $p$-Value & $\beta$ & SE & $p$-Value \\
\hline Women vs. men & 6.9 & 2.6 & 0.008 & 6.4 & 2.9 & 0.029 & 3.9 & 2.6 & 0.138 \\
\hline Age group vs. 40 s or older & & & $<0.001 \mathrm{~d}$ & & & $<0.001 \mathrm{~d}$ & & & $<0.001 \mathrm{~d}$ \\
\hline $20 \mathrm{~s}$ & 13 & 2.0 & & 12 & 2.1 & & 8.4 & 2.1 & \\
\hline $30 \mathrm{~s}$ & 6.4 & 2.4 & & 6.9 & 2.7 & & 3.8 & 2.5 & \\
\hline Marital status (Married vs. Single) & -11 & 1.7 & $<0.001$ & -11 & 2.0 & $<0.001$ & -6.7 & 1.8 & $<0.001$ \\
\hline Presence of a child & -12 & 2 & $<0.001$ & -11 & 2.1 & $<0.001$ & -9.1 & 1.8 & $<0.001$ \\
\hline Housekeeping hours in weekdays & -3.5 & 1.8 & 0.048 & -3.0 & 2.0 & 0.133 & -3.3 & 1.8 & 0.062 \\
\hline Housekeeping hours in weekend & -3.8 & 1.7 & 0.028 & -2.7 & 2.0 & 0.169 & -4.3 & 1.7 & 0.014 \\
\hline WFC & 2.2 & 0.2 & $<0.001$ & 2.4 & 0.21 & $<0.001$ & 1.9 & 0.19 & $<0.001$ \\
\hline Working experience (years) & -9.2 & 1.7 & $<0.001$ & -8.7 & 1.9 & $<0.001$ & -5.1 & 1.7 & 0.004 \\
\hline Work hours & 1.9 & 0.56 & $<0.001$ & 2.8 & 0.63 & $<0.001$ & 2.0 & 1.6 & $<0.001$ \\
\hline Working nights per month & 6.4 & 1.7 & $<0.001$ & 5.7 & 1.9 & 0.003 & 8.2 & 1.7 & $<0.001$ \\
\hline High strain vs. Low strain & 13 & 1.7 & $<0.001$ & 16 & 1.9 & $<0.001$ & 10 & 1.8 & $<0.001$ \\
\hline High support vs. Low support & -8.7 & 1.7 & $<0.001$ & -8.5 & 1.9 & $<0.001$ & -5.4 & 1.7 & 0.002 \\
\hline Physical symptoms (Reference: none) & & & $<0.001 \mathrm{~d}$ & & & $<0.001 \mathrm{~d}$ & & & $<0.001 \mathrm{~d}$ \\
\hline $1-2$ & 9.2 & 2.2 & & 15 & 2.4 & & 7.1 & 2.3 & \\
\hline $3-4$ & 15 & 2.3 & & 21 & 2.6 & & 6.9 & 2.5 & \\
\hline $5-$ & 25 & 2.3 & & 35 & 2.5 & & 18 & 2.4 & \\
\hline \multicolumn{10}{|c|}{ Individual models for three particular symptoms } \\
\hline Fatigue & 19 & 1.5 & $<0.001$ & 26 & 1.6 & $<0.001$ & 11 & 1.7 & $<0.001$ \\
\hline Backache & 11 & 1.7 & $<0.001$ & 16 & 1.9 & $<0.001$ & 11 & 1.7 & $<0.001$ \\
\hline Insomnia & 16 & 2.0 & $<0.001$ & 17 & 2.3 & $<0.001$ & 9.1 & 2.1 & $<0.001$ \\
\hline
\end{tabular}

d trend $\mathrm{P}$

WBO: work-related burnout PBO: personal burnout CBO: client-related burnout WFC: work-family conflict.

Table 6 shows multivariable general linear models for factors associated with WBO, $\mathrm{PBO}$ and $\mathrm{CBO}$ among hospital workers. The WBO score increased with the quartile of physical symptoms $(+7.9$ points for $1-2$ symptoms; +11 points for $3-4$ symptoms; 18 points for 5 or more symptoms; $p<0.001)$, WFC $(+1.7$ points, $p<0.001)$, and job strain $(+4.6$ points, $p=0.003)$. The WBO decreased when the participants had a child $(-7.6$ points, $p=0.001)$ and job support $(-4.7$ points, $p=0.001)$. When either fatigue, backache, or insomnia was entered into the model instead of physical symptom quartile, each symptom was significantly associated with an increased risk of WBO (all $p$ values $<0.001$ ), among which fatigue was most strongly correlated with $\mathrm{WBO}(+13$ points, $p<0.001)$. 
Table 6. Multivariable general linear models of an effect of covariates according to burnout subscale among hospital workers.

\begin{tabular}{|c|c|c|c|c|c|c|c|c|c|}
\hline & \multicolumn{3}{|c|}{ WBO } & \multicolumn{3}{|c|}{ PBO } & \multicolumn{3}{|c|}{ СВO } \\
\hline & \multicolumn{3}{|c|}{$(n=466, \mathrm{R} 247 \%)$} & \multicolumn{3}{|c|}{$(n=466, \mathrm{R} 249 \%)$} & \multicolumn{3}{|c|}{$(n=466, \mathrm{R} 232 \%)$} \\
\hline & $\beta$ & SE & $p$-Value & $\beta$ & SE & $p$-Value & $\beta$ & SE & $p$-Value \\
\hline Women vs. Men & 2.9 & 2.2 & 0.195 & 1.5 & 2.5 & 0.545 & 2.1 & 2.5 & 0.415 \\
\hline Age group vs. 40 s or older & & & $0.297 \mathrm{~d}$ & & & $0.032 \mathrm{~d}$ & & & $0.764 \mathrm{~d}$ \\
\hline 0 - 0 - 1 & 8.2 & 3.4 & & 7.4 & 3.9 & & 3.2 & 4 & \\
\hline $30 \mathrm{~s}$ & 3.4 & 2.1 & & 4.4 & 2.3 & & -1.0 & 2.4 & \\
\hline Marital status (Married vs. Single) & -6.5 & 2.1 & 0.435 & -2.9 & 2.3 & 0.205 & 1.3 & 2.4 & 0.593 \\
\hline Presence of a child & -7.6 & 2.3 & 0.001 & -5.3 & 2.6 & 0.040 & -7.7 & 2.6 & 0.004 \\
\hline Housekeeping hours in weekdays & -0.93 & 1.8 & 0.611 & -0.87 & 2.1 & 0.674 & -1.2 & 2.1 & 0.555 \\
\hline Housekeeping hours in weekend & -0.41 & 1.8 & 0.823 & 1.0 & 2.0 & 0.634 & -1.6 & 2.1 & 0.447 \\
\hline WFC & 1.7 & 0.17 & $<0.001$ & 1.6 & 0.19 & $<0.001$ & 1.7 & 0.20 & $<0.001$ \\
\hline Working experience & 1.7 & 3.0 & 0.567 & 0.41 & 3.4 & 0.905 & 3.2 & 3.5 & 0.354 \\
\hline Work hours & 0.27 & 0.48 & 0.574 & 1.2 & 0.54 & 0.025 & 0.42 & 0.55 & 0.444 \\
\hline Working nights per month & 0.70 & 1.5 & 0.645 & -0.63 & 1.7 & 0.711 & 4.1 & 1.7 & 0.019 \\
\hline High strain vs. Low strain & 4.6 & 1.5 & 0.003 & 7.3 & 1.7 & $<0.001$ & 3.1 & 1.8 & 0.079 \\
\hline High support vs. Low support & -4.7 & 1.4 & 0.001 & -2.7 & 1.6 & 0.097 & -2.6 & 1.7 & 0.117 \\
\hline Physical symptoms (Reference: none) & & & $<0.001 \mathrm{~d}$ & & & $<0.001 \mathrm{~d}$ & & & $<0.001 \mathrm{~d}$ \\
\hline $1-2$ & 7.9 & 2.0 & & 14 & 2.2 & & 6.8 & 2.3 & \\
\hline $3-4$ & 11 & 2.2 & & 18 & 2.5 & & 3.8 & 2.5 & \\
\hline $5-$ & 18 & 2.2 & & 28 & 2.4 & & 13 & 2.5 & \\
\hline \multicolumn{10}{|l|}{ Individual models for three particular symptoms } \\
\hline Fatigue a & 13 & 1.5 & $<0.001$ & 20 & 1.7 & $<0.001$ & 5.6 & 1.8 & 0.002 \\
\hline Backache b & 6.0 & 1.5 & $<0.001$ & 10 & 1.8 & $<0.001$ & 7.0 & 1.7 & $<0.001$ \\
\hline Insomnia c & 11 & 1.8 & $<0.001$ & 12 & 2.1 & $<0.001$ & 6.1 & 2.0 & 0.003 \\
\hline \multicolumn{10}{|l|}{ 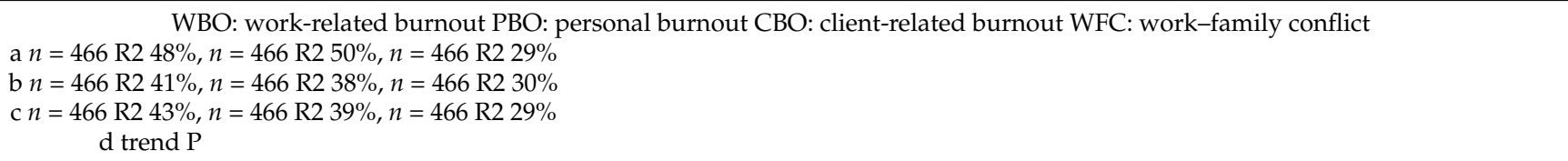 } \\
\hline
\end{tabular}

The PBO score increased with the quartile of physical symptoms (+14 points for $1-2$ symptoms; +18 points for $3-4$ symptoms; +28 points for 5 or more symptoms; $p<0.001$ ), younger age group $(+7.4$ points in $20 \mathrm{~s}$, and +4.4 in $30 \mathrm{~s}, p=0.032)$, WFC $(+1.6$ points, $p<0.001)$, work hours $(+1.2$ points per one hour increase, $p=0.025)$, and job strain $(+7.3$ points, $p<0.001)$. The PBO score decreased when they had a child $(-5.3$ points, $p=0.040)$. When either fatigue, backache, or insomnia was entered into the model instead of physical symptom quartile, each symptom was significantly associated with an increased risk of PBO (all $p$ values $<0.001$ ), among which fatigue was most strongly correlated with $\mathrm{PBO}(+20$ points, $p<0.001)$.

The CBO score increased with quartile of physical symptoms (+6.8 points for $1-2$ symptoms; +3.8 points for $3-4$ symptoms; 13 points for 5 or more symptoms, $p<0.001$ ), WFC ( +1.7 points, $p<0.001)$, more working nights per month $(+4.1$ points, $p=0.019)$ while the score decreased when the participant had a child $(-7.7$ points, $p=0.004)$. When either fatigue, backache, or insomnia was entered into the model instead of physical symptom quartile, each symptom was significantly associated with an increased risk of $\mathrm{CBO}$ ( $p$ values $=0.002,<0.001$, and 0.003 , respectively), among which backache was most strongly correlated with $\mathrm{CBO}(+7.0$ points, $p<0.001)$.

\section{Discussion}

This study found that the perception of more physical symptoms was significantly and linearly associated with higher burnout scores in all three Copenhagen burnout subscales, with one exception: CBO among faculties. Overall, job strain and WFC were associated with an increased risk of burnout, which may increase intention to leave their profession, as suggested by previous studies [29,30]. In addition, we demonstrated that being married (WBO and $\mathrm{CBO}$ among faculties), having a child (except for $\mathrm{PBO}$ and $\mathrm{CBO}$ among faculties), 
and job support (faculty and hospital workers with WBO and faculties with PBO) were associated with a decreased risk of burnout, suggesting that social support from family members and colleagues at work may mitigate burnout levels.

In this study, we focused on the number of physical symptoms, rather than the individual symptoms themselves. Previous studies have demonstrated that psychological stress has a marked impact on physical symptoms, including fatigue [31], bowel changes (diarrhea and constipation) [32], insomnia [33], and chronic pain or discomfort [34]. It sometimes results in a life-threatening disease, such as depression and its attendant risk of suicide or self-harm [35] or coronary heart disease [36]. Indeed, our study confirmed that the most frequent symptoms-fatigue and backache, as well as insomnia-were independently associated with burnout, except for $\mathrm{CBO}$ among faculties. Furthermore, it should be noted that the beta estimate of fatigue was almost equivalent to those of three or four physical symptoms in all of the CBI subscales. This result may be interpreted to mean that multiple physical symptoms could be useful to identify burnout if there are more than five physical symptoms. Indeed, referring to the Diagnostic and Statistical Manual of Mental Disorders, Fifth Edition, fatigue is a symptom of major depression; therefore, it is well known to be highly correlated with psychological distress, including burnout. Our study results are unique in that they show that a greater number of perceived physical symptoms, rather than fewer symptoms, was associated with higher degrees (i.e., beta estimates) of burnout.

It is often difficult for an employee to report a mental health problem to their employer, as reporting it may directly cause job termination. The most frequently reported mistreatment in the workplace due to reporting job stress is an unwanted lay-off, followed by fewer opportunities for promotion and salary increase. In Japan, the Labor Contract Act prohibits employers from forcing the termination of an employee for medical reasons, such as depression, without considering the possibility of recovery; however, it still occurs [37]. In these circumstances when maltreatment/discrimination occurs, workers do not consult with the industrial doctors directly hired by the hospitals they work for because they believe that those industrial doctors are linked with the employer. The Japan Labor Safety and Hygiene Law introduced a mental health screening program (i.e., Act 66) at periodic health checkups in 2015; however, some workers are not willing to take the tests and tell their employer the truth. The situation is more serious among medical professionals because they understand that reporting a mental health problem to their employer may create additional disadvantages. In this regard, the results of the present study are useful, as physical symptoms might serve as a warning of an employee's mental health status.

Among the three types of burnout, our multivariable regression model demonstrated that physical symptoms were not associated with $\mathrm{CBO}$ among faculties. In our study, burnout scores of the three domains were smaller among faculties than among hospital workers. The discrepancy in burnout levels between the two professions may be due to labor characteristics. Most hospital workers in our participant group are nurses, who are primarily responsible for the psychological and emotional aspects of patients associated with illnesses, while faculties take care of students rather than patients. The discrepancy may be explained by the "client" (patients for hospital workers and students for faculties) each professional takes care of, with patients being more demanding in clinical settings than students.

Inconsistent results have been reported in the past on the effect of having a child as a mitigating or risk factor for burnout. Due to gender roles in traditional Japanese society and the traditional division of labor augmenting WFC among high-achieving professionals, having a child has long been believed to cause career obstacles among professional women [38]. However, having a child functioned as a mitigating factor of burnout in our study, despite the presence of "work-life conflict" [39]. When children are in early childhood, parents are more likely to commit to child-rearing, which might affect WFC. Although our dataset does not include the age of participants' children, considering the participants' average age (38 years), their children might be grown enough to be more 
independent, which may have a buffering effect on the correlation between having children and burnout. Previously, Sarason et al. [40]. indicated that perceived social support can affect an individual's emotional well-being. A previous study similarly demonstrated that social support factors from "family" and "friends" have consistently shown the strongest associations with symptomatology among clinically distressed people and a student sample [41]. The significant and independent effect of having a child, apart from marital status and other social support, suggests the possible buffering effect of having a child on burnout.

Our study had several limitations. First, the response rate for our survey was low, at $31 \%$, which may result in nonresponse bias. Second, because this study was embedded in a periodic university survey about work-life balance conducted by a women's support center, women were more likely to participate from both source populations of faculties (women's response rate $88 \%$ : women constitute $20 \%$ of 1137 faculty) and hospital workers (women's response rate $42 \%$ : women constitute $48 \%$ of 2629 healthcare professionals). In contrast, those who face greater work demands (and may be at higher risk of burnout as a result) may have opted out, which might lead to an underestimation. Third, our study population was derived from the faculties of one private university, indicating that generalizability may be limited to some extent. Fourth, unmeasured confounding factors may still have existed, even if we had made an effort to include personal factors along with working conditions to explain burnout.

Although our results require careful interpretation due to these limitations, we believe that our study is valid in its conclusion that a greater number of physical symptoms perceived could indicate burnout. The practical implication may include the various physical symptoms in the periodical health check-up questionnaire. Those who manifested five or more physical symptoms perceived more often than once a week may be at risk of burnout. Thus, the number of physical symptoms may be a useful indicator to identify a high-risk individual who may require early intervention.

\section{Conclusions}

Individuals with more physical symptoms had higher burnout scores on all three CBI subscales, except for $\mathrm{CBO}$ among faculties, even after adjusting for potential confounding variables. As employees may find physical symptoms easier or more socially acceptable to report than mental health issues, it is feasible to count the number of symptoms and incorporate them into the periodic health checkup questionnaire. This may help to identify individuals at risk of burnout. The number of physical symptoms may become an easy, simple, and useful indicator to identify high-risk individuals in the workplace, particularly those in the medical field, who are more likely to experience burnout.

Author Contributions: Conceptualization, K.N.; methodology, K.N.; software, H.H.; validation, H.O.; formal analysis, Y.C.; investigation, K.N. and A.T.; resources, H.O.; data curation, A.T.; writingoriginal draft preparation, Y.C.; writing-review and editing, K.N.; visualization, K.N.; supervision, K.N.; project administration, H.O.; funding acquisition, K.N. All authors have read and agreed to the published version of the manuscript.

Funding: Nomura is funded by the Ministry of Education, Culture, Sports, Science and Technology, Japan (Grants for Scientific Research [B], Number 16H0526).

Institutional Review Board Statement: The study was conducted according to the guidelines of the Declaration of Helsinki and approved by the by the Institutional Review Board of Teikyo University School of Medicine (\#TEIRIN 15-141 in 2015).

Informed Consent Statement: Informed consent was obtained from all subjects involved in the study.

Data Availability Statement: Our data are available upon reasonable request.

Acknowledgments: Chithra R. Perumalswami helped us in revising this article with useful suggestions and insights.

Conflicts of Interest: The authors declare no conflict of interest. 


\section{References}

1. Nakao, M.; Yano, E. Reporting of somatic symptoms as a screening marker for detecting major depression in a population of Japanese white-collar workers. J. Clin. Epidemiol. 2003, 56, 1021-1026. [CrossRef]

2. Isshiki, A.; Nakao, M.; Yamaoka, K.; Yano, E. Application of symptom checklist for screening major depression by annual health examinations: A cross-validity study in the workplace. J. Med. Screen. 2004, 11, 207-209. [CrossRef] [PubMed]

3. Bauer, A.M.; Chen, C.-N.; Alegría, M. Associations of physical symptoms with perceived need for and use of mental health services among Latino and Asian Americans. Soc. Sci. Med. 2012, 75, 1128-1133. [CrossRef]

4. Lee, P.P.; Cunningham, W.E.; Nakazono, T.T.; Hays, R.D. Associations of Eye Diseases and Symptoms with Self-Reported Physical and Mental Health. Am. J. Ophthalmol. 2009, 148, 804-808.e1. [CrossRef]

5. Maslach, C.J.S.; Leiter, M. Maslach Burnout Inventory Manual, 3rd ed.; Consulting Psychologists: Palo Alto, CA, USA, 1996.

6. Salari, N.; Khazaie, H.; Hosseinian-Far, A.; Khaledi-Paveh, B.; Kazeminia, M.; Mohammadi, M.; Shohaimi, S.; Daneshkhah, A.; Eskandari, S. The prevalence of stress, anxiety and depression within front-line healthcare workers caring for COVID-19 patients: A systematic review and meta-regression. Hum. Resour. Health 2020, 18, 1-14. [CrossRef]

7. North, N.; Leung, W.; Ashton, T.; Rasmussen, E.; Hughes, F.; Finlayson, M. Nurse turnover in New Zealand: Costs and relationships with staffing practises and patient outcomes. J. Nurs. Manag. 2012, 21, 419-428. [CrossRef]

8. Tei-Tominaga, M. Factors related to the intention to leave and the decision to resign among newly graduated nurses: A complete survey in a selected prefecture in Japan. Environ. Health Prev. Med. 2013, 18, 293-305. [CrossRef]

9. Lacy, B.E.; Chan, J.L. Physician Burnout: The Hidden Health Care Crisis. Clin. Gastroenterol. Hepatol. 2018, 16, 311-317. [CrossRef]

10. Rothenberger, D.A. Physician Burnout and Well-Being: A Systematic Review and Framework for Action. Dis. Colon Rectum 2017, 60, 567-576. [CrossRef] [PubMed]

11. Kelly, L.A.; Lefton, C.; Fischer, S.A. Nurse Leader Burnout, Satisfaction, and Work-Life Balance. JONA J. Nurs. Adm. 2019, 49, 404-410. [CrossRef] [PubMed]

12. Boamah, S.A.; Read, E.A.; Laschinger, H.K.S. Factors influencing new graduate nurse burnout development, job satisfaction and patient care quality: A time-lagged study. J. Adv. Nurs. 2017, 73, 1182-1195. [CrossRef] [PubMed]

13. Alves, P.C.; Oliveira, A.D.F.; Paro, H.B.M.D.S. Quality of life and burnout among faculty members: How much does the field of knowledge matter? PLoS ONE 2019, 14, e0214217. [CrossRef] [PubMed]

14. Nomura, K.; Nakao, M.; Sato, M.; Ishikawa, H.; Yano, E. The association of the reporting of somatic symptoms with job stress and active coping among Japanese white-collar workers. J. Occup. Health 2007, 49, 370-375. [CrossRef] [PubMed]

15. Karasek, R.; Brisson, C.; Kawakami, N.; Houtman, I.; Bongers, P.; Amick, B. The Job Content Questionnaire (JCQ): An instrument for internationally comparative assessments of psychosocial job characteristics. J. Occup. Health Psychol. 1998, 3, 322-355. [CrossRef] [PubMed]

16. Gold, K.J.; Andrew, L.B.; Goldman, E.B.; Schwenk, T.L. I would never want to have a mental health diagnosis on my record": A survey of female physicians on mental health diagnosis, treatment, and reporting. Gen. Hosp. Psychiatry 2016, $43,51-57$. [CrossRef] [PubMed]

17. Kristensen, T.S.; Borritz, M.; Villadsen, E.; Christensen, K.B. The Copenhagen Burnout Inventory: A new tool for the assessment of burnout. Work. Stress 2005, 19, 192-207. [CrossRef]

18. Borritz, M.; Kristensen, T.S. Copenhagen Burnout Inventory; Normative Data from a Representative Danish Population on Personal Burnout and Results from the PUMA* Study on Personal Burnout, Work Burnout, and Client Burnout; National Institute of Occupational Health: Copenhagen, Denmark, 2004.

19. Hall, L.H.; Johnson, J.; Watt, I.; Tsipa, A.; O'Connor, D.B. Healthcare Staff Wellbeing, Burnout, and Patient Safety: A Systematic Review. PLoS ONE 2016, 11, e0159015. [CrossRef] [PubMed]

20. Borritz, M.; Rugulies, R.; Bjorner, J.B.; Villadsen, E.; Mikkelsen, O.A.; Kristensen, T.S. Burnout among employees in human service work: Design and baseline findings of the PUMA study. Scand. J. Public Health 2006, 34, 49-58. [CrossRef]

21. Borritz, M.; Christensen, K.B.; Bültmann, U.; Rugulies, R.; Lund, T.; Andersen, I.; Villadsen, E.; Diderichsen, F.; Kristensen, T.S. Impact of Burnout and Psychosocial Work Characteristics on Future Long-Term Sickness Absence. Prospective Results of the Danish PUMA Study Among Human Service Workers. J. Occup. Environ. Med. 2010, 52, 964-970. [CrossRef]

22. Nakao, M.; Yamanaka, G.; Kuboki, T. Suicidal ideation and somatic symptoms of patients with mind/body distress in a Japanese psychosomatic clinic. Suicide Life-Threat. Behav. 2002, 32, 80-90. [CrossRef]

23. Kroenke, K. Physical symptoms in primary care. Predictors of psychiatric disorders and functional impairment. Arch. Fam. Med. 1994, 3, 774-779. [CrossRef] [PubMed]

24. Greenhaus, J.H.; Parasuraman, S. Career involvement and family involvement as moderators of relationships between workfamily conflict and withdrawal from a profession. J. Occup. Health Psychol. 2001, 6, 91-100. [CrossRef]

25. Millings, A.; Hirst, S.L.; Sirois, F.; Houlston, C. Emotional adaptation to relationship dissolution in parents and non-parents: A new conceptual model and measure. PLoS ONE 2020, 15, e239712. [CrossRef] [PubMed]

26. Watai, I.; Nishikido, N.; Murashima, S. Japanese Scale of Work-Family Conflict, Development and Discussion. J. Occupational. Health 2006, 48, 71-81.

27. Kawakami, N.; Kobayashi, F.; Araki, S.; Haratani, T.; Furui, H. Assessment of job stress dimensions based on the job demandscontrol model of employees of telecommunication and electric power companies in japan: Reliability and validity of the japanese version of the job content questionnaire. Int. J. Behav. Med. 1995, 2, 358-375. [CrossRef] 
28. Li, L.; Wu, C.; Gan, Y.; Qu, X.; Lu, Z. Insomnia and the risk of depression: A meta-analysis of prospective cohort studies. BMC Psychiatry 2016, 16, 1-16. [CrossRef]

29. Van Der Heijden, B.; Mahoney, C.B.; Xu, Y. Impact of Job Demands and Resources on Nurses' Burnout and Occupational Turnover Intention Towards an Age-Moderated Mediation Model for the Nursing Profession. Int. J. Environ. Res. Public Health 2019, $16,2011$. [CrossRef]

30. Yedidia, M.J.; Chou, J.; Brownlee, S.; Flynn, L.; Tanner, C.A. Association of Faculty Perceptions of Work-Life with Emotional Exhaustion and Intent to Leave Academic Nursing: Report on a National Survey of Nurse Faculty. J. Nurs. Educ. 2014, 53, 569-579. [CrossRef] [PubMed]

31. Rose, D.M.; Seidler, A.; Nübling, M.; Latza, U.; Brähler, E.; Klein, E.M.; Wiltink, J.; Michal, M.; Nickels, S.; Wild, P.S.; et al. Associations of fatigue to work-related stress, mental and physical health in an employed community sample. BMC Psychiatry 2017, 17, 167. [CrossRef]

32. Qin, H.-Y.; Cheng, C.-W.; Tang, X.-D.; Bian, Z.-X. Impact of psychological stress on irritable bowel syndrome. World J. Gastroenterol. 2014, 20, 14126-14131. [CrossRef]

33. Metlaine, A.; Sauvet, F.; Gomez-Merino, D.; Elbaz, M.; Delafosse, J.Y.; Leger, D.; Chennaoui, M. Association between insomnia symptoms, job strain and burnout syndrome: A cross-sectional survey of 1300 financial workers. BMJ Open 2017, 7, e012816. [CrossRef]

34. Kopec, J.A.; Sayre, E.C. Work-related psychosocial factors and chronic pain: A prospective cohort study in Canadian workers. J. Occup. Environ. Med. 2004, 46, 1263-1271. [PubMed]

35. Deeb, G.R.; Braun, S.; Carrico, C.; Kinser, P.; Laskin, D.; Deeb, J.G. Burnout, depression and suicidal ideation in dental and dental hygiene students. Eur. J. Dent. Educ. 2017, 22, e70-e74. [CrossRef] [PubMed]

36. Tofler, G.H.; Kopel, E.; Klempfner, R.; Eldar, M.; Buckley, T.; Goldenberg, I. Triggers and Timing of Acute Coronary Syndromes. Am. J. Cardiol. 2017, 119, 1560-1565. [CrossRef]

37. Legal Consultation about Firing. Available online: https://rodosoudan.net/blog-entry-4.html (accessed on 25 January 2020).

38. Petek, D.; Gajsek, T.; Ster, M.P. Work-family balance by women GP specialist trainees in Slovenia: A qualitative study. BMC Med. Educ. 2016, 16, 31. [CrossRef] [PubMed]

39. Chatani, Y.; Nomura, K.; Horie, S.; Takemoto, K.; Takeuchi, M.; Sasamori, Y.; Takenoshita, S.; Murakami, A.; Hiraike, H.; Okinaga, H.; et al. Effects of gaps in priorities between ideal and real lives on psychological burnout among academic faculty members at a medical university in Japan: A cross-sectional study. Environ. Health Prev. Med. 2017, 22, 1-7. [CrossRef] [PubMed]

40. Sarason, I.G.; Sarason, B.R. Concomitants of social support: Attitudes, personality characteristics, and life experiences. J. Pers. 1982, 50, 331-344. [CrossRef] [PubMed]

41. Clara, I.; Cox, B.; Enns, M.; Murray, L.; Torgrudc, L. Confirmatory Factor Analysis of The Multidimensional Scale of Perceived Social Support in Clinically Distressed and Student Samples. J. Pers. Assess. 2003, 81, 265-270. [CrossRef] [PubMed] 